\title{
SILICON CARBIDE GRAINS OF TYPE C PROVIDE EVIDENCE FOR THE PRODUCTION OF THE UNSTABLE ISOTOPE ${ }^{32} \mathrm{Si}$ IN SUPERNOVAE
}

\author{
M. Pignatari ${ }^{1,14}$, E. Zinner ${ }^{2}$, M. G. Bertolli ${ }^{3,14}$, R. Trappitsch ${ }^{4,5}{ }^{14}$, P. Hoppe $^{6}$, T. Rauscher $^{1,7}$, C. Fryer $^{8,14}$, \\ F. Herwig ${ }^{9,10,14}$, R. Hirschi ${ }^{11,12,14}$, F. X. Timmes ${ }^{10,13,14}$, AND F.-K. ThielemanN ${ }^{1}$ \\ ${ }^{1}$ Department of Physics, University of Basel, Klingelbergstrasse 82, CH-4056 Basel, Switzerland \\ ${ }^{2}$ Laboratory for Space Sciences and the Physics Department, Washington University, St. Louis, MO 63130, USA \\ ${ }^{3}$ Theoretical Division (T-2), LANL, Los Alamos, NM 87545, USA \\ ${ }^{4}$ Department of the Geophysical Sciences, University of Chicago, Chicago, IL 60637, USA \\ ${ }^{5}$ Chicago Center for Cosmochemistry, University of Chicago, Chicago, IL 60637, USA \\ ${ }^{6}$ Max Planck Institute for Chemistry, Hahn-Meitner-Weg 1, D-55128 Mainz, Germany \\ ${ }^{7}$ Centre for Astrophysics Research, School of Physics, Astronomy and Mathematics, University of Hertfordshire, College Lane, Hatfield AL10 9AB, UK \\ ${ }^{8}$ Computational Physics and Methods (CCS-2), LANL, Los Alamos, NM 87545, USA \\ ${ }^{9}$ Department of Physics and Astronomy, University of Victoria, Victoria, BC, Canada \\ ${ }^{10}$ The Joint Institute for Nuclear Astrophysics, Notre Dame, IN 46556, USA \\ ${ }^{11}$ Keele University, Keele, Staffordshire ST5 5BG, UK \\ ${ }^{12}$ Kavli Institute for the Physics and Mathematics of the Universe (WPI), University of Tokyo, 5-1-5 Kashiwanoha, Kashiwa 277-8583, Japan \\ ${ }^{13}$ School of Earth and Space Exploration, Arizona State University, P.O. Box 871404, Tempe, AZ 85287-1404, USA \\ Received 2013 March 30; accepted 2013 May 23; published 2013 June 13
}

\begin{abstract}
Carbon-rich grains are observed to condense in the ejecta of recent core-collapse supernovae (SNe) within a year after the explosion. Silicon carbide grains of type X are C-rich grains with isotopic signatures of explosive SN nucleosynthesis have been found in primitive meteorites. Much rarer silicon carbide grains of type $\mathrm{C}$ are a special sub-group of $\mathrm{SiC}$ grains from $\mathrm{SNe}$. They show peculiar abundance signatures for $\mathrm{Si}$ and $\mathrm{S}$, isotopically heavy $\mathrm{Si}$, and isotopically light $\mathrm{S}$, which appear to be in disagreement with model predictions. We propose that $\mathrm{C}$ grains are formed mostly from $\mathrm{C}$-rich stellar material exposed to lower SN shock temperatures than the more common type $\mathrm{X}$ grains. In this scenario, extreme ${ }^{32} \mathrm{~S}$ enrichments observed in $\mathrm{C}$ grains may be explained by the presence of short-lived ${ }^{32} \mathrm{Si}\left(\tau_{1 / 2}=153 \mathrm{yr}\right)$ in the ejecta, produced by neutron capture processes starting from the stable $\mathrm{Si}$ isotopes. No mixing from deeper Si-rich material and/or fractionation of Si from S due to molecular chemistry is needed to explain the ${ }^{32} \mathrm{~S}$ enrichments. The abundance of ${ }^{32} \mathrm{Si}$ in the grains can provide constraints on the neutron density reached during the SN explosion in the C-rich He shell material. The impact of the large uncertainty of the neutron capture cross sections in the ${ }^{32} \mathrm{Si}$ region is discussed.
\end{abstract}

Key words: stars: abundances - stars: evolution - stars: interiors - stars: massive

Online-only material: color figures

\section{INTRODUCTION}

Despite recent improvements in simulations of core-collapse supernova (CCSN) explosions (e.g., Janka 2012), the understanding of supernova ( $\mathrm{SN})$ still has major gaps, and observations of SN and their ejecta still provide many puzzles (e.g., Fryer et al. 2012 and references therein). Of particular importance may be the asymmetric nature of the explosion and the hydrodynamic development of the layers ejected after the explosion (e.g., Kjær et al. 2010; Isensee et al. 2010; DeLaney et al. 2010).

Several types of presolar grains from primitive carbonaceous meteorites that are associated with SN nucleosynthesis due to their isotopic ratios (see, e.g., Clayton \& Nittler 2004; Zinner 2007) provide constraints on these explosions. Presolar grains carry the signatures of their stellar origin, and their interpretation may help to guide CCSN models.

Silicon carbide is one of the types of stardust grains that have been identified in primitive meteorites (e.g., Zinner 2007). While most of these so-called presolar $\mathrm{SiC}$ grains originate in asymptotic giant branch stars, there are two rare sub-types of $\mathrm{SiC}$ grains that have a CCSN origin. Type X grains (about $1 \%$ of all presolar $\mathrm{SiC}$ grains) have large excesses in ${ }^{28} \mathrm{Si}$. This signature

\footnotetext{
${ }^{14}$ NuGrid Collaboration, http://www.nugridstars.org
}

and evidence for the initial presence of ${ }^{44} \mathrm{Ti}$ in a subset of these grains is proof of their $\mathrm{SN}$ origin: both isotopes are predicted to be abundant in the $\mathrm{Si} / \mathrm{S}$ zone of SNe (Rauscher et al. 2002). More recently, Pignatari et al. (2013, hereafter P13), showed that ${ }^{28} \mathrm{Si}$ and ${ }^{44} \mathrm{Ti}$ may also be produced at the bottom of the $\mathrm{He}$ shell exposed to high shock velocities and/or high energies, reproducing several isotopic abundance patterns typical of $\mathrm{SiC} \mathrm{X}$ grains and graphites from $\mathrm{SNe}$.

Silicon carbide grains of type $\mathrm{C}$ are even rarer (about $0.1 \%$ of all $\mathrm{SiC}$ grains) than $\mathrm{SiC} \mathrm{X}$ grains. They have a large excess in ${ }^{29} \mathrm{Si}$ and ${ }^{30} \mathrm{Si}$ and most of them have been found by automatic searches in the NanoSIMS detection apparatus. Some of these grains contain extinct ${ }^{44} \mathrm{Ti}$, similar to $\mathrm{SiC} \mathrm{X}$ grains. Just over a dozen of these grains have been identified, and 9 have been analyzed for their $\mathrm{S}$ isotopic ratios, showing large ${ }^{32} \mathrm{~S}$ excesses, with ${ }^{32} \mathrm{~S} / 33,34$ S ratios ranging up to 16 times solar (Amari et al. 1999; Croat et al. 2010; Gyngard et al. 2010; Hoppe et al. 2010, 2012; Zinner et al. 2010; Orthous-Daunay et al. 2012; Xu et al. 2012). This is puzzling because in existing SN models the only zone with large ${ }^{32} \mathrm{~S}$ excesses is the $\mathrm{Si} / \mathrm{S}$ zone (Meyer et al. 1995), which has large ${ }^{28} \mathrm{Si}$ excesses, whereas zones with ${ }^{28} \mathrm{Si}$ depletions (i.e., ${ }^{29,30} \mathrm{Si}$ excesses) are predicted to have also ${ }^{32} \mathrm{~S}$ depletions (e.g., Rauscher et al. 2002). Hoppe et al. (2012) have invoked element fractionation between sulfur and silicon by molecule chemistry in the SN ejecta to explain 


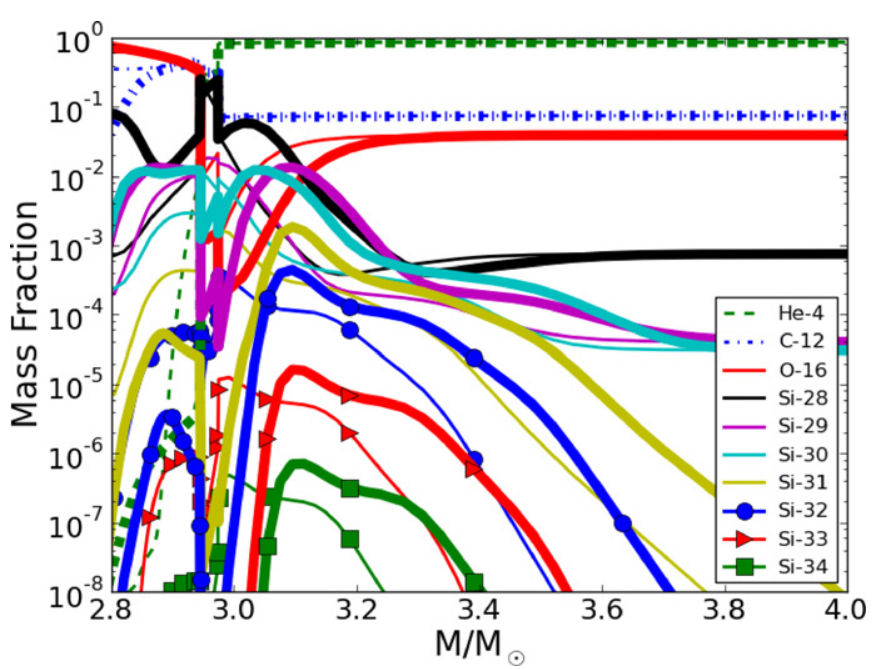

Figure 1. Isotopic abundance profiles given $2.5 \mathrm{~s}$ after the explosion in the top of the $\mathrm{O} / \mathrm{C}$ zone, the $\mathrm{C} / \mathrm{Si}$ zone, and the $\mathrm{He} / \mathrm{C}$ zone of the $15 M_{\odot} \mathrm{SN}$ models $15 \mathrm{r}$ and $15 \mathrm{r} 4$. Shown are profiles for ${ }^{4} \mathrm{He},{ }^{12} \mathrm{C},{ }^{16} \mathrm{O}$, and the $\mathrm{Si}$ isotopes along the neutron capture chain from ${ }^{28} \mathrm{Si}$ to ${ }^{34} \mathrm{Si}$. The models $15 \mathrm{r}$ and $15 \mathrm{r} 4$ are represented by thick and thin lines, respectively.

(A color version of this figure is available in the online journal.)

this result. However, this ad hoc explanation cannot explain all the data, especially the $\mathrm{S}$ isotopic composition of one $\mathrm{C}$ grain with $\delta\left({ }^{33} \mathrm{~S} /{ }^{32} \mathrm{~S}\right)$ and $\delta\left({ }^{34} \mathrm{~S} /{ }^{32} \mathrm{~S}\right)$ values being as low as $-940 \%$ o (Xu et al. 2012), even more extreme than those of $\mathrm{S}$ in the $\mathrm{Si} / \mathrm{S}$ zone. In this Letter, we propose that the ${ }^{32} \mathrm{~S}$ excesses in $\mathrm{C}$ grains are due to the radioactive decay of short-lived ${ }^{32} \mathrm{Si}$ $\left(\tau_{1 / 2}=153 \mathrm{yr}\right.$; Ouellet \& Balraj 2011). We present models of explosive nucleosynthesis in the inner part of the $\mathrm{He} / \mathrm{C}$ zone, where ${ }^{29} \mathrm{Si}$ and ${ }^{30} \mathrm{Si}$ as well as ${ }^{32} \mathrm{~S}$ excesses can be produced while maintaining a $\mathrm{C}$-rich environment.

The Letter is organized as follows. In Section 2 we describe the stellar models and the nucleosynthesis calculations, in Section 3 we compare theoretical results with measurements for $\mathrm{C}$ grains. Finally, in Section 4 we give our conclusions.

\section{STELLAR MODEL CALCULATIONS AND NUCLEOSYNTHESIS}

This investigation is based on seven SN explosion models for a $15 M_{\odot}, Z=0.02$ star, three of which were introduced in P13. The pre-SN evolution is calculated with the code GENEC (Eggenberger et al. 2008). The explosion simulations include the fallback prescription by Fryer et al. (2012), and are performed for a case with recommended initial shock velocity and six cases where the latter is reduced by a factor of 2, 4, 5, 10, 20, and 100 , respectively (models $15 \mathrm{r}, 15 \mathrm{r} 2,15 \mathrm{r} 4,15 \mathrm{r} 5,15 \mathrm{r} 10,15 \mathrm{r} 20$, and 15r100). The standard initial shock velocity used beyond fallback is $2 \times 10^{9} \mathrm{~cm} \mathrm{~s}^{-1}$. The kinetic explosion energy for these $15 M_{\odot}$ models ranges from 4 to $5 \times 10^{51} \mathrm{erg}$ to less than $10^{51} \mathrm{erg}$. The post-processing code MPPNP is used to calculate the nucleosynthesis in the star before and during the explosion (see, e.g., Bennett et al. 2012). In the present study, we focus only on the C-rich explosive He-burning layers, including the $\mathrm{He} / \mathrm{C}$ zone and a small part of the $\mathrm{O} / \mathrm{C}$ zone.

The abundances of key species and ${ }^{28-34} \mathrm{Si}$ are reported in Figure 1 for models $15 \mathrm{r}$ and $15 \mathrm{r} 4$. Results are similar for the intermediate model $15 \mathrm{r} 2$. The bottom of the $\mathrm{He} / \mathrm{C}$ zone is strongly affected by the explosion. While ${ }^{12} \mathrm{C}$ is not significantly modified, ${ }^{16} \mathrm{O}$ is depleted and feeds the production of heavier
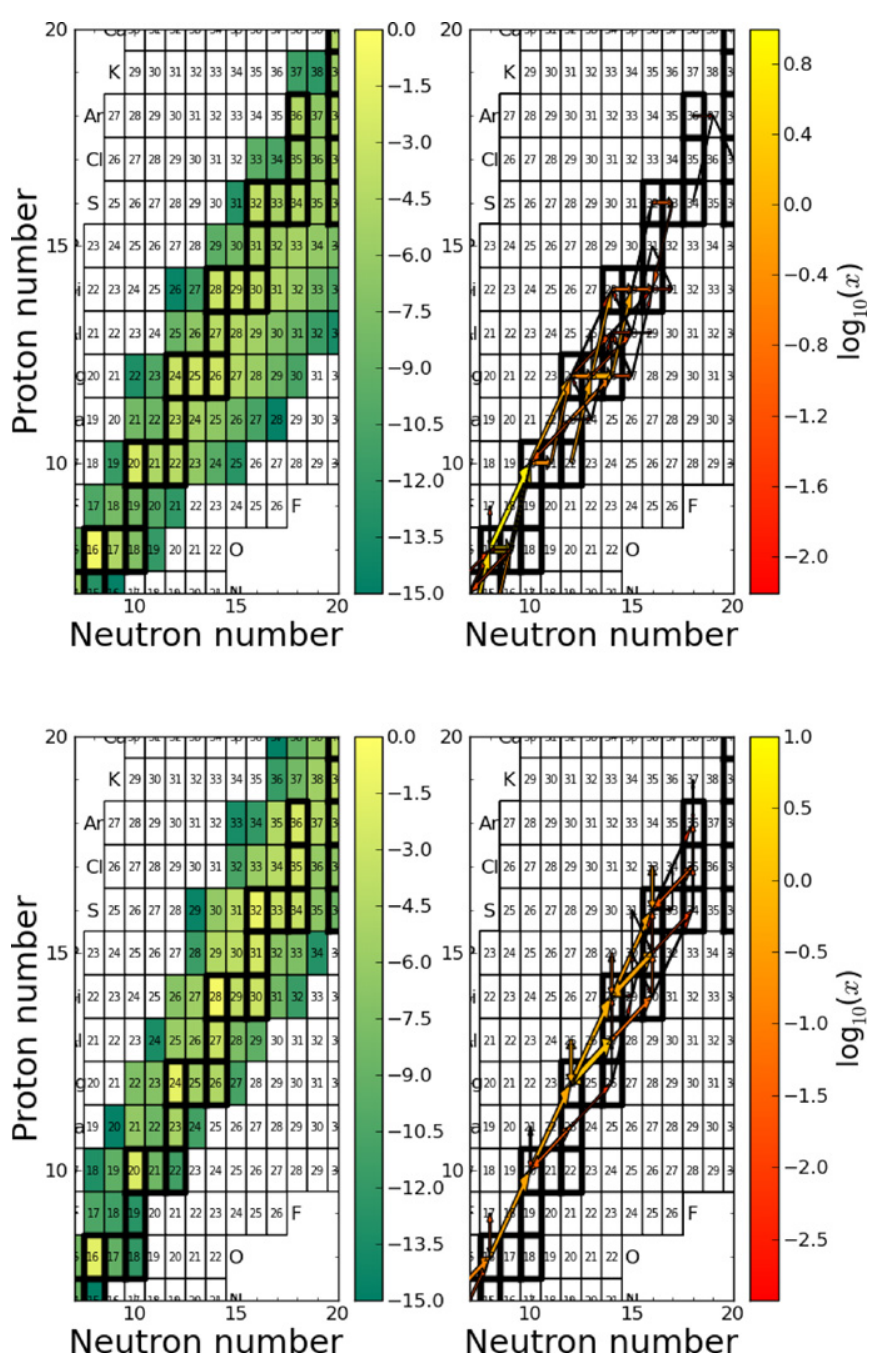

Figure 2. Mass fraction abundance distributions (green, left) and nucleosynthesis fluxes (arrows with red to yellow color, right) at $\sim 10^{-5} \mathrm{~s}$ (upper panels) and $\sim 10^{-2} \mathrm{~s}$ (lower panels) after the explosion at mass coordinate $2.95 M_{\odot}$ of the model $15 \mathrm{r}$. The nucleosynthesis fluxes, $\left[\delta Y_{\mathrm{i}} / \delta t\right]_{\mathrm{j}}$, show the variation of the abundance $Y_{\mathrm{i}}=X_{\mathrm{i}} / A_{\mathrm{i}}$ due to the reaction $\mathrm{j}$. The arrow width and color correspond to the flux strength. Heavy-lined boxes correspond to the stable isotopes.

(A color version of this figure is available in the online journal.)

$\alpha$-isotopes, including ${ }^{28} \mathrm{Si}$. This stellar region was defined as the $\mathrm{C} / \mathrm{Si}$ zone in $\mathrm{P} 13$. The main reason for this behavior is the higher $\alpha$-capture rates starting from the ${ }^{16} \mathrm{O}(\alpha, \gamma){ }^{20} \mathrm{Ne}$ reaction than that of the ${ }^{12} \mathrm{C}(\alpha, \gamma){ }^{16} \mathrm{O}$ reaction at explosive He shell temperatures (as explained by P13). Models with lower shock velocities show weaker explosion signatures. In particular, model 15r100 does not show any significant departures from pre-explosive abundances during the explosion in the C-rich region.

Along the Si neutron capture chain, ${ }^{29-30} \mathrm{Si}$ and heavier unstable Si species are produced efficiently by neutron captures starting from ${ }^{28} \mathrm{Si}$. The larger explosion temperatures in model $15 \mathrm{r}$ than in model $15 \mathrm{r} 4$ are pushing the production peaks of different $\mathrm{Si}$ neutron-rich species to larger mass coordinates, not significantly affecting their absolute abundance. Therefore, abundance yields for the $\mathrm{Si}$ isotopes in the explosive He shell result from the interplay between $\alpha$-captures and neutron captures, triggered by activation of the ${ }^{22} \mathrm{Ne}(\alpha, n)^{25} \mathrm{Mg}$ neutron source (e.g., Meyer et al. 2000 and references therein). The main abundance features and dominating nucleosynthesis fluxes for two different times of the SN explosion are given in Figure 2, in the so-called $\mathrm{C} / \mathrm{Si}$ zone $\left(M \sim 2.95 M_{\odot}\right.$, model $15 \mathrm{r}$; see also 

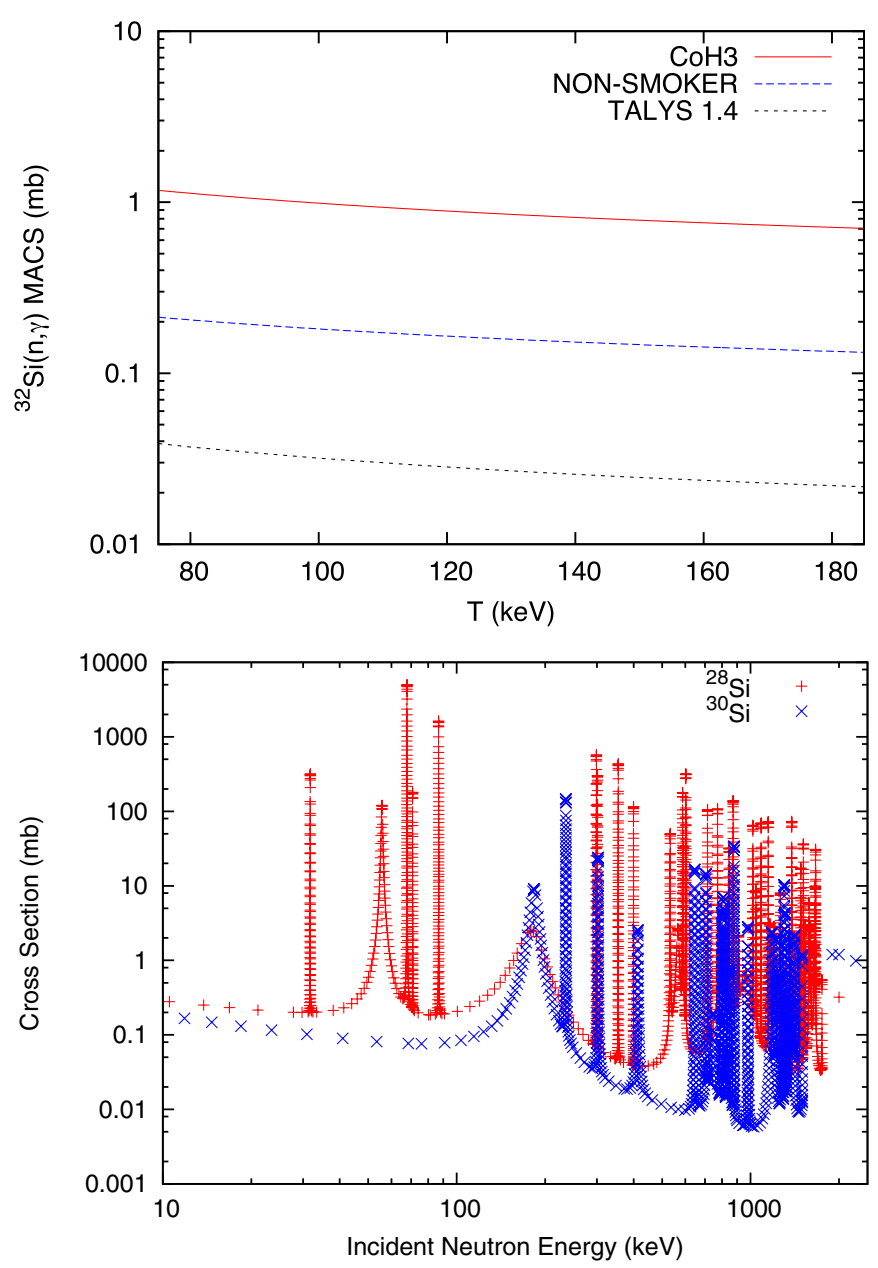

Figure 3. Upper panel: neutron capture MACS for ${ }^{32} \mathrm{Si}$, calculated by different statistical HF models in the temperature range of interest $T=1-2 \times 10^{9} \mathrm{~K}$ (corresponding to $\sim 90-170 \mathrm{keV}$ ). No experimental data exist for ${ }^{32} \mathrm{Si}$, therefore one has to rely on theoretical calculations. Statistical methods are not applicable in the primary energy range of interest for this nucleus, therefore a more appropriate approach is needed to constrain the uncertainty. Lower panel: neutron capture cross sections for ${ }^{28} \mathrm{Si}$ and ${ }^{30} \mathrm{Si}$ from the ENDF/B-VII.1 library (see the end of Section 2 for discussion).

(A color version of this figure is available in the online journal.)

P13). In the early stages of the explosion, depending on the available ${ }^{22} \mathrm{Ne}$, the $\alpha$-capture path starting from ${ }^{16} \mathrm{O}$ is accompanied by $(n, \gamma)(\alpha, n)$ sequences, producing the same $\alpha$-species. An example is $\left.{ }^{20} \mathrm{Ne}(\alpha, \gamma)\right)^{24} \mathrm{Mg}$ and $\left.{ }^{20} \mathrm{Ne}(n, \gamma)\right)^{21} \mathrm{Ne}(\alpha, n)^{24} \mathrm{Mg}$. During the later stages of the explosion and/or low ${ }^{22} \mathrm{Ne}$ abundances, the $(\alpha, \gamma)$ fluxes become dominant. Note that for explosive He-burning conditions the $(\alpha, p)$ fluxes are compensated by their reverse reactions, and proton captures on the abundant $\alpha$-isotopes do not affect the abundance of their parent species because of the efficient reverse $(\gamma, p)$ photodisintegrations.

In the present calculations, we use for the $(n, \gamma)$ reactions on unstable $\mathrm{Si}$ species the rates from Hauser-Feshbach (HF) calculations by the NON-SMOKER code (Rauscher \& Thielemann 2000), available in JINA REACLIB version 1.1 (e.g., Cyburt et al. 2010). The uncertainties of the neutron capture rates in the mass region of ${ }^{32} \mathrm{Si}$ are very large. Figure 3, upper panel, shows the Maxwellian-averaged cross section (MACS) for neutron capture on ${ }^{32} \mathrm{Si}$ as calculated from the $\mathrm{HF}$ codes $\mathrm{CoH}_{3}$ (Kawano et al. 2004) and TALYS 1.4 (Koning et al. 2008, 2011), and NON-SMOKER. At a temperature near $90 \mathrm{keV}\left(\sim 10^{9} \mathrm{~K}\right)$, we see a difference of almost two orders of magnitude between the highest (from $\mathrm{CoH}_{3}$ ) and the lowest calculated values (TALYS 1.4). Note, however, that these theoretical predictions are still consistent within the large uncertainty of the ${ }^{32} \mathrm{Si}(n, \gamma)^{33} \mathrm{Si}$ rate. The large uncertainty is due to the nuclear level density being too low to apply the HF model for neutron-rich isotopes of $\mathrm{Si}$. The model relies on the statistical averaging over levels in the compound nucleus and thus a sufficiently high nuclear level density is required at the compound formation energy (Rauscher et al. 1997). The ENDF/B-VII.1 library of Chadwick et al. (2011) provides the location of neutron capture resonances for even-even nuclei near ${ }^{32} \mathrm{Si}$, as shown in Figure 3, lower panel. While no data are available on ${ }^{32} \mathrm{Si}$ neutron capture resonances, the neighboring even-even nuclei ${ }^{30} \mathrm{Si}$ and ${ }^{28} \mathrm{Si}$ give an indication of the number of levels accessible at different incident particle energies. Above an energy of $\sim 600 \mathrm{keV}$ statistical methods become appropriate. For this reason, we considered an uncertainty of a factor of 100 for the ${ }^{32} \mathrm{Si}$ neutron capture cross section. The impact of this uncertainty is presented in Section 3.

Where experimental knowledge of the single resonances has been obtained, such as in the case of ${ }^{28} \mathrm{Si}$ and ${ }^{30} \mathrm{Si}$, uncertainties may still arise from the precise location and strength of each resonance. However, uncertainties from experiment are expected to be much lower than those introduced by the use of HF calculations in an inappropriate region.

\section{COMPARISON WITH OBSERVATIONS}

We compare in Figure 4 the abundances from the $\mathrm{C}$-rich ejecta from our models (Section 2) originating from the $\mathrm{C} / \mathrm{Si}$ zone, the whole $\mathrm{He} / \mathrm{C}$ zone, and the $\mathrm{C}$-rich part of the $\mathrm{He} / \mathrm{N}$ zone, with isotopic ratios of single $\mathrm{SiC} \mathrm{X}$ and $\mathrm{C}$ grains from the St. Louis Presolar Grains Database (Hynes \& Gyngard 2009). No mixing between layers is considered and $\mathrm{SiC} \mathrm{X}$ and $\mathrm{C}$ grains with ${ }^{12} \mathrm{C} /{ }^{13} \mathrm{C}$ lower than solar are excluded. They are not reproduced by these models that have high $\mathrm{C}$ isotopic ratio.

The standard model (15r, upper panel, layer 1 Figure 4) shows a strong ${ }^{28} \mathrm{Si}$ and ${ }^{32} \mathrm{~S}$ production and the absence of ${ }^{32} \mathrm{Si}$ in the $\mathrm{C} / \mathrm{Si}$ zone during the explosion (see also P13). Outward, in the inner part of the $\mathrm{He} / \mathrm{C}$ zone, the lower explosion temperatures and the neutron burst triggered by the ${ }^{22} \mathrm{Ne}(\alpha, n)^{25} \mathrm{Mg}$ ( $n$ process; e.g., Meyer et al. 2000) gradually reduce ${ }^{28} \mathrm{Si}$ and ${ }^{32} \mathrm{~S}$ enrichments, whereas ${ }^{32} \mathrm{Si}$ is synthesized and accumulated according to its neutron capture cross section (as discussed in Section 2). The outer parts of the $\mathrm{He} / \mathrm{C}$ zone show mild enrichments of the stable neutron-rich $\mathrm{Si}$ and $\mathrm{S}$ isotopes due to pre-explosive $s$-processing.

The ${ }^{28} \mathrm{Si}$ excess observed in $\mathrm{SiC} \mathrm{X}$ grains are reproduced in parts of the $\mathrm{C} / \mathrm{Si}$ zone for the models $15 \mathrm{r}$ and $15 \mathrm{r} 2$ (e.g., layer 1 of model 15r, Figure 4, lower panel). SiC C grains show larger ${ }^{32} \mathrm{~S}$ excesses than $\mathrm{SiC} \mathrm{X}$ grains, and positive $\delta\left({ }^{30} \mathrm{Si}\right)$. Such a signature is consistent with abundance predictions from more external zones in the C-rich He shell. In models 15r4-15r20, the shock temperature is not sufficient to reproduce the ${ }^{28} \mathrm{Si}$ excess observed in SiC X grains (see also P13). Conversely, the presented models can reproduce the $\mathrm{Si}$ and $\mathrm{S}$ isotopic ratios in the $\mathrm{C}$ grains over a large range in initial shock velocities. Also in the case of contamination or mixing with isotopically more normal material (see P13), the grain signatures can be explained since $\delta\left({ }^{30} \mathrm{Si}\right)$ values up to $\sim 15,000-20,000$ (e.g., models $15 \mathrm{r}$, $15 \mathrm{r} 2$, and $15 \mathrm{r} 4$, zones " 2 " and " 3 ") are associated with large ${ }^{32} \mathrm{~S}$ enrichments $\left(\delta\left({ }^{34} \mathrm{~S}\right) \sim-1000\right.$, Figure 4 , lower panel, outside the plot range). For most of the He shell material, the ${ }^{32} \mathrm{Si}$ signature dominates $\mathrm{S}$ isotopic anomalies, assuming an arbitrary $\mathrm{Si} / \mathrm{S}$ 

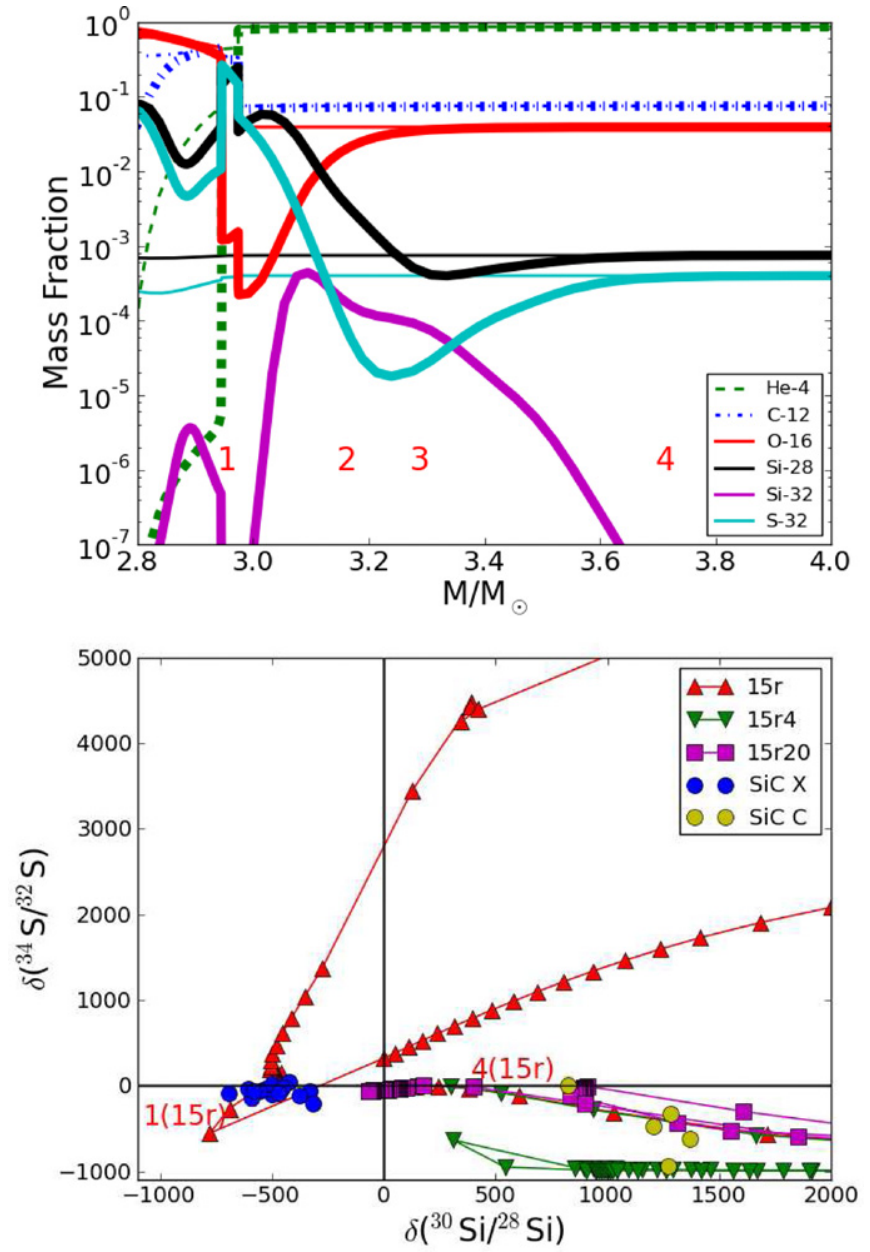

Figure 4. Upper panel: abundances of selected isotopes in the outer part of the $\mathrm{O} / \mathrm{C}$ zone and the $\mathrm{He} / \mathrm{C}$ zone for model $15 \mathrm{r}$ before and after CCSN explosion (thin and thick lines). Lower panel: the ${ }^{30} \mathrm{Si} /{ }^{28} \mathrm{Si}$ and ${ }^{34} \mathrm{~S} /{ }^{32} \mathrm{~S}$ isotopic ratios of presolar $\mathrm{SiC} \mathrm{X}$ and $\mathrm{C}$ grains, plotted as $\delta$-values, deviations from the solar ratios in parts per thousand (\%o), are compared with predictions of three different models $(15 r, 15 r 4$, and $15 r 20)$ in the mass range shown in the upper panel. We highlight the predicted $\mathrm{Si}$ and $\mathrm{S}$ isotopic ratios of model $15 \mathrm{r}$ at two different mass coordinates, $M=2.95$ and $3.7 M_{\odot}$ (see upper panel). The $\mathrm{Si}$ isotopic ratios of zones " 2 " and "3" are located out of the plot range, with $\delta\left({ }^{30} \mathrm{Si}\right) \sim$ 15,000 and $\delta\left({ }^{34} \mathrm{~S}\right) \sim-1000$. A fractionation factor of $10^{4} \mathrm{for} \mathrm{Si} / \mathrm{S}$ is assumed. (A color version of this figure is available in the online journal.)

fractionation of $10^{4}$ during grain formation (Figure 4, lower panel). This assumption expresses the hypothesis that all ${ }^{32} \mathrm{~S}$ observed in $\mathrm{C}$ grains originates from the decay of ${ }^{32} \mathrm{Si}$ (see below for details). Only little $\mathrm{S}$ condenses into $\mathrm{SiC}$ grains, justifying the assumed elemental fractionation (e.g., Amari et al. 1995).

In Figure 5, upper panel, we show the ${ }^{32} \mathrm{Si} /{ }^{28} \mathrm{Si}$ isotopic ratios from different models described in Section 2, comparing them with the ratios inferred for $\mathrm{C}$ grains from the radiogenic ${ }^{32} \mathrm{~S}$. We estimated the ratio of the radioactive ${ }^{32} \mathrm{Si}\left({ }^{32} \mathrm{~S}^{*}\right)$ to ${ }^{28} \mathrm{Si}$ and thus the original ${ }^{32} \mathrm{Si} /{ }^{28} \mathrm{Si}$ ratio by assuming that all the $\mathrm{S}\left(\mathrm{S}_{\mathrm{tot}}\right)$ in the grains was either ${ }^{32} \mathrm{~S}^{*}$ or isotopically normal $\mathrm{S}\left(\mathrm{S}_{\text {norm }}\right)$ from contamination. The latter assumption is based on the fact that $\mathrm{S}$ is volatile and is not likely to condense into $\mathrm{SiC}$. The grains are therefore expected to contain only marginal intrinsic $\mathrm{S}$. Second, the $\mathrm{S}$ concentrations are low in the He shell layers with no ${ }^{28} \mathrm{Si}$ enrichment. Finally, some of the $\mathrm{S}$ isotopic images of the $\mathrm{C}$ grains measured showed ${ }^{34} \mathrm{~S}$ to be more abundant at the edges of the grains and ${ }^{32} \mathrm{~S}$ excesses to be higher in interior
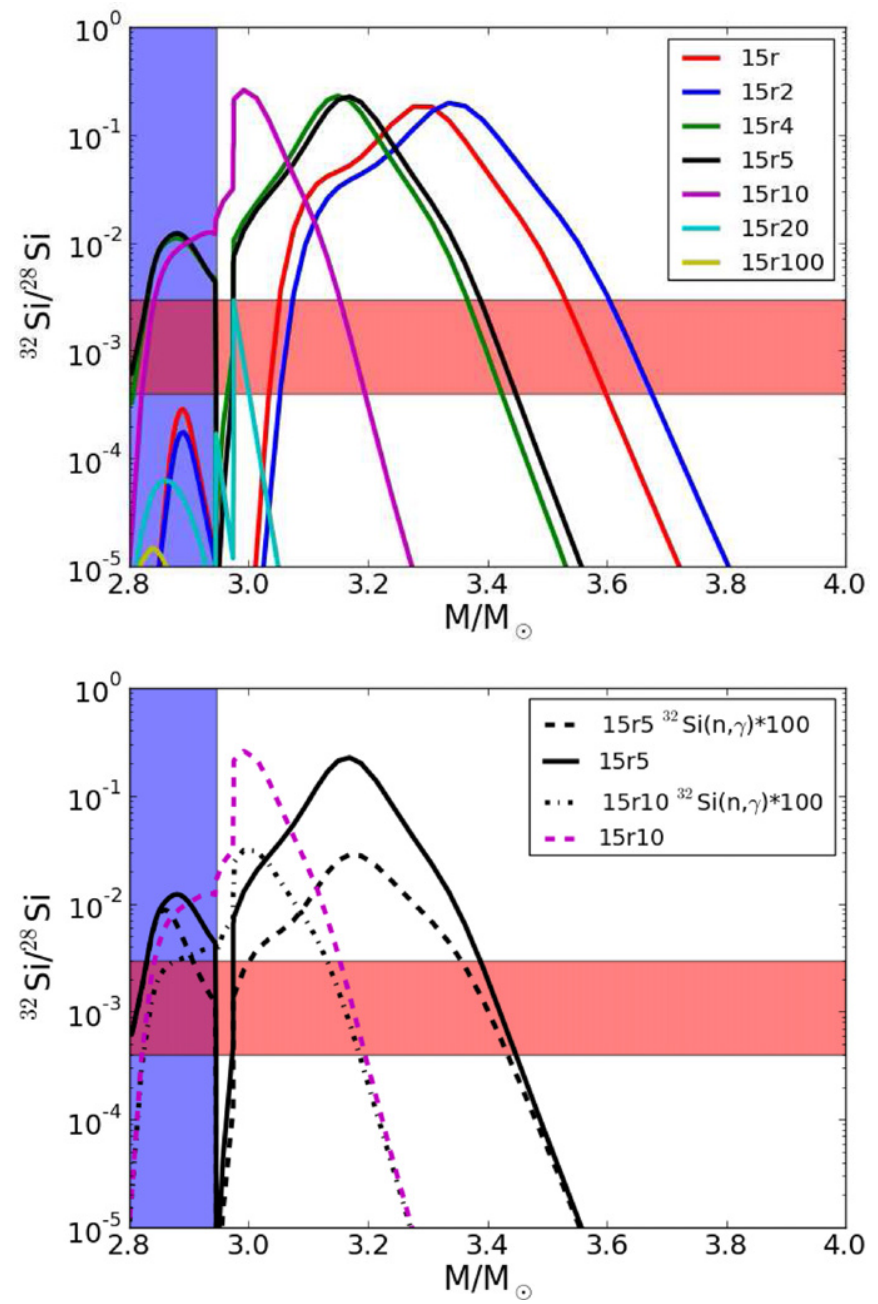

Figure 5. Upper panel: final isotopic ratio ${ }^{32} \mathrm{Si} /{ }^{28} \mathrm{Si}$ in the C-rich explosive He shell for models with initial shock velocities varying by a factor of 100 (decreasing from model $15 \mathrm{r}$ to $15 \mathrm{r} 100$ ). The blue-shaded area denotes O-rich material. For comparison, the red-shaded area indicates the range of ${ }^{32} \mathrm{Si} /{ }^{28} \mathrm{Si}$ ratios, inferred from $\mathrm{S}$ isotopic ratios and $\mathrm{S}$ and $\mathrm{Si}$ abundances in presolar $\mathrm{SiC}$ $\mathrm{C}$ grains, under the assumption that the measured ${ }^{32} \mathrm{~S}$ excess derives from ${ }^{32} \mathrm{Si}$ decay. Lower panel: impact on the ${ }^{32} \mathrm{Si} /{ }^{28} \mathrm{Si}$ ratio from increasing the ${ }^{32} \mathrm{Si}$ neutron capture cross section by a factor of 100 for models $15 \mathrm{r} 5$ and $15 \mathrm{r} 10$.

(A color version of this figure is available in the online journal.)

than in border regions. We determined the atomic ${ }^{32} \mathrm{Si} /{ }^{28} \mathrm{Si}$ ratios by applying an $\mathrm{S}^{-} / \mathrm{Si}^{-}$sensitivity factor of three, inferred from measurements of $\mathrm{Si}$ and $\mathrm{S}$ ion yields on synthetic $\mathrm{SiC}$ and Mundrabilla FeS, respectively (Hoppe et al. 2012). Since ${ }^{32} \mathrm{~S}_{\text {tot }}={ }^{32} \mathrm{~S}^{*}+{ }^{32} \mathrm{~S}_{\text {norm }}$ and ${ }^{32} \mathrm{~S}^{*}=-0.001 \times \delta \mathrm{S} \times\left({ }^{32} \mathrm{~S}^{*}+{ }^{32} \mathrm{~S}_{\text {norm }}\right)$, we obtained the ${ }^{32} \mathrm{~S}^{*} /{ }^{28} \mathrm{Si}$ ratios by multiplying ${ }^{32} \mathrm{~S} /{ }^{28} \mathrm{Si}$ with $-0.001 \times \delta \mathrm{S}$. Here, ${ }^{32} \mathrm{~S}_{\text {norm }}$ is ${ }^{32} \mathrm{~S}$ of the isotopically normal component $\mathbf{S}_{\text {norm }}$ (assumed to be contamination). For $\delta \mathrm{S}$, we took the average of $\delta\left({ }^{33} \mathrm{~S} /{ }^{32} \mathrm{~S}\right)$ and $\delta\left({ }^{34} \mathrm{~S} /{ }^{32} \mathrm{~S}\right)$. Within errors the latter two values are equal for all measured grains, providing additional evidence that we are dealing just with an excess in ${ }^{32} \mathrm{Si}$. In Figure 5, we show that the observed range of ${ }^{32} \mathrm{Si} /{ }^{28} \mathrm{Si}$ ratios is matched by predictions from stellar models at different energies, in agreement with Figure 4. Typical conditions required for matching the inferred ${ }^{32} \mathrm{Si} /{ }^{28} \mathrm{Si}$ ratios (e.g., at $M=3.4 M_{\odot}$ for models $15 \mathrm{r} 4$ and $15 \mathrm{r} 5$ ) have a peak temperature of $\sim 8 \times 10^{8} \mathrm{~K}$ and a neutron density peak of $\sim 10^{18-19} \mathrm{~cm}^{-3}$, with a ${ }^{28} \mathrm{Si}$ mass fraction of $\sim 5 \times 10^{-4}$. The 
models $15 \mathrm{r}-15 \mathrm{r} 5$ with the highest explosion temperatures also fit the observed ${ }^{32} \mathrm{Si} /{ }^{28} \mathrm{Si}$ ratio deeper in the He shell (e.g., at $M=3.05 M_{\odot}$ for models $15 \mathrm{r}$ and $15 \mathrm{r} 2$ ), with a ${ }^{28} \mathrm{Si}$ mass fraction of $\sim 5 \times 10^{-2}$. In these cases, the temperature peak is about $1.6 \times 10^{9} \mathrm{~K}$, with a neutron density peak of a few $10^{22} \mathrm{~cm}^{-3}$ for few $10^{-5} \mathrm{~s}$, dropping quickly to densities more typical of the $n$ process.

Since the grains may contain some normal component (P13), the inferred ${ }^{32} \mathrm{Si} /{ }^{28} \mathrm{Si}$ needs to be considered a lower limit of the original ratio in the He shell material. In Figure 5, lower panel, we show that increasing the neutron capture cross section of ${ }^{32} \mathrm{Si}$ by a factor of 100 (see discussion in Section 2) does not change our results. By reducing the ${ }^{32} \mathrm{Si}$ MACS of the same factor the ${ }^{32} \mathrm{Si} /{ }^{28} \mathrm{Si}$ ratio increases by less than $10 \%$, since the ${ }^{32} \mathrm{Si}$ MACS adopted in our models is already lower than $1 \mathrm{mb}$, behaving as a bottleneck in the neutron capture flow feeding heavier Si species. Note that at the temperatures of explosive He burning the half-life of ${ }^{32} \mathrm{Si}$ can be reduced down to few days (e.g., Oda et al. 1994). However, the timescale of the explosive nucleosynthesis is less than $\sim 0.3 \mathrm{~s}$, and the impact of the ${ }^{32} \mathrm{Si}$ half-life in the calculations is negligible.

We have shown that CCSN models can explain the large ${ }^{32} \mathrm{~S}$ excess measured in $\mathrm{SiC} \mathrm{C}$ grains by the radioactive decay of the unstable isotope ${ }^{32} \mathrm{Si}$ after grain formation. Furthermore, in $\mathrm{SiC} \mathrm{C}$ grains most of the remaining $\mathrm{S}$ is coming from contamination. We have identified two typical conditions where the correct ${ }^{32} \mathrm{Si} /{ }^{28} \mathrm{Si}$ ratio can be obtained, depending on the explosion temperature and on the abundance of ${ }^{28} \mathrm{Si}$.

\section{CONCLUSIONS}

We have compared the isotopic signatures in presolar $\mathrm{SiC}$ grains of type $\mathrm{C}$ with nucleosynthesis predictions for CCSN ejecta exposed to different shock velocities. We propose that the seemingly incompatible $\mathrm{Si}$ and $\mathrm{S}$ isotopic ratios in these grains are explained by assuming that the ${ }^{32} \mathrm{~S}$ excess observed today originates from radioactive ${ }^{32} \mathrm{Si}$ that condensed into the forming $\mathrm{SiC}$ grains, and decayed into ${ }^{32} \mathrm{~S}$ at later stages. Assuming that all the remaining $\mathrm{S}$ is due to contamination, we estimated the ${ }^{32} \mathrm{Si} /{ }^{28} \mathrm{Si}$ ratio in the parent $\mathrm{CCSN}$ ejecta, ranging from a few $10^{-4}$ to a few $10^{-3}$. We propose this ratio to be a lower limit of its original value in the explosive He shell layers, depending on the level of contamination or mixing with more normal material for each $\mathrm{C}$ grain. Such ratios can be produced for different shock velocities and/or explosion energies. Two typical conditions reproducing directly the observed ${ }^{32} \mathrm{Si} /{ }^{28} \mathrm{Si}$ ratios are: one with high temperature and large ${ }^{28} \mathrm{Si}$ abundance $\left(\sim 1.6 \times 10^{9} \mathrm{~K}\right.$ and $\sim 5 \times 10^{-2}$, respectively), and the other with temperature $\sim 0.7-0.9 \times 10^{9} \mathrm{~K}$ and ${ }^{28} \mathrm{Si}$ abundance $\sim 5 \times 10^{-4}$. In the first case, the neutron density reaches a peak of a few $10^{22} \mathrm{~cm}^{-3}$ for few $10^{-5} \mathrm{~s}$, rapidly dropping to values more typical of the $n$-process neutron burst. In the second case, the neutron density peak is on the order of $10^{18-19} \mathrm{~cm}^{-3}$.

In conclusion, $\mathrm{C}$ grains carry a record of the neutron density reached in the explosive He shell of the CCSN where they formed. We showed that the theoretical nuclear reactions in the ${ }^{32} \mathrm{Si}$ mass region have large uncertainties, but our results are not significantly affected. We conclude that $\mathrm{C}$ grains carry the signature of lower energy ejecta compared to $\mathrm{SiC} X$ grains, showing positive $\delta(\mathrm{Si})$ values and a significant amount of ${ }^{32} \mathrm{Si}$ produced by neutron captures.
We thank the anonymous referee for the very careful review of this Letter, which significantly contributed to improving the quality of the publication. NuGrid acknowledges significant support from NSF grants PHY 02-16783 and PHY 09-22648 (Joint Institute for Nuclear Astrophysics, JINA) and EU MIRGCT-2006-046520. The continued work on codes and in disseminating data is made possible through funding from STFC and EU-FP7-ERC-2012-St Grant 306901 (R.H., UK), and NSERC Discovery grant (F.H., Canada), and an Ambizione grant of the SNSF (M.P., Switzerland). M.P., T.R., R.H., and F.K.T. also thank for support from EuroGENESIS. NuGrid data are served by Canfar/CADC. E.Z. was supported by NASA grant NNX11AH14G. M.G.B.'s research was carried out under the auspices of the National Nuclear Security Administration of the U.S. Department of Energy at Los Alamos National Laboratory under Contract No. DE-AC52-06NA25396. P.H. thanks Ramanath Cowsik for his hospitality at the McDonnell Center for the Space Sciences at Washington University. T.R. also acknowledges the support from the THEXO Collaboration within the EU Seventh Framework Program, the European Research Council, and the Swiss NSF. R.H. also acknowledges support from the World Premier International Research Center Initiative (WPI Initiative), MEXT, Japan, and from European Research Council under the European Union's Seventh Framework Program (FP/2007-2013)/ERC Grant Agreement No. 306901.

\section{REFERENCES}

Amari, S., Lewis, R. S., \& Anders, E. 1995, GeCoA, 59, 1411

Amari, S., Zinner, E., \& Lewis, R. S. 1999, ApJL, 517, L59

Bennett, M. E., Hirschi, R., Pignatari, M., et al. 2012, MNRAS, 420, 3047 Chadwick, M., Herman, M., Obložinský, P., et al. 2011, NDS, 112, 2887

Clayton, D. D., \& Nittler, L. R. 2004, ARA\&A, 42, 39

Croat, T. K., Stadermann, F. J., \& Bernatowicz, T. J. 2010, AJ, 139, 2159

Cyburt, R. H., Amthor, A. M., Ferguson, R., et al. 2010, ApJS, 189, 240

DeLaney, T., Rudnick, L., Stage, M. D., et al. 2010, ApJ, 725, 2038

Eggenberger, P., Meynet, G., Maeder, A., et al. 2008, Ap\&SS, 316, 43

Fryer, C. L., Belczynski, K., Wiktorowicz, G., et al. 2012, ApJ, 749, 91

Gyngard, F., Nittler, L. R., \& Zinner, E. 2010, M\&PSA, 73, 5242

Hoppe, P., Fujiya, W., \& Zinner, E. 2012, ApJL, 745, L26

Hoppe, P., Leitner, J., Gröner, E., et al. 2010, ApJ, 719, 1370

Hynes, K. M., \& Gyngard, F. 2009, in 40th Lunar and Planetary Institute Science Conference, 1198

Isensee, K., Rudnick, L., DeLaney, T., et al. 2010, ApJ, 725, 2059

Janka, H.-T. 2012, ARNPS, 62, 407

Kawano, T., Chiba, S., Maruyama, T., et al. 2004, in Proc. 2003 Symp. on Nuclear Data, ed. T. Ohsawa \& T. Fukahori (Tokai: Japan Atomic Energy Research Institute), 196

Kjær, K., Leibundgut, B., Fransson, C., Jerkstrand, A., \& Spyromilio, J. 2010, A\&A, 517, A51

Koning, A., Hilaire, S., \& Goriely, S. 2011, TALYS-1.4 (Petten, The Netherlands: Nuclear Research and Consultancy Group)

Koning, A. J., Hilaire, S., \& Duijvestijn, M. C. 2008, in Proc. Int. Conf. on Nuclear Data for Science and Technology, ed. O. Bersillon, F. Gunsing, E. Bauge, R. Jacqmin, \& S. Leray (Les Ulis: EDP Sciences), 211

Meyer, B. S., Clayton, D. D., \& The, L.-S. 2000, ApJL, 540, L49

Meyer, B. S., Weaver, T. A., \& Woosley, S. E. 1995, Metic, 30, 325

Oda, T., Hino, M., Muto, K., Takahara, M., \& Sato, K. 1994, ADNDT, 56, 231

Orthous-Daunay, F.-R., Gyngard, F., Moynier, F., \& Zinner, E. 2012, in 43rd Lunar and Planetary Institute Science Conference, 2679

Ouellet, C., \& Balraj, B. 2011, NDS, 112, 2199

Pignatari, M., Wiescher, M., Timmes, F. X., et al. 2013, ApJL, 767, L22

Rauscher, T., Heger, A., Hoffman, R. D., \& Woosley, S. E. 2002, ApJ, 576, 323 Rauscher, T., \& Thielemann, F.-K. 2000, ADNDT, 75, 1

Rauscher, T., Thielemann, F. K., \& Kratz, K.-L. 1997, PhRvC, 56, 1613

Xu, Y. C., Amari, S., Gyngard, F., Zinner, E., \& Lin, Y. 2012, M\&PSA, 47,5104

Zinner, E. 2007, in Treatise on Geochemistry, Update 1.02, ed. H. D. Holland, K. K. Turekian, \& A. Davis (Oxford: Elsevier), 1

Zinner, E., Gyngard, F., \& Nittler, L. R. 2010, in 41st Lunar and Planetary Institute Science Conference, 1359 